NASA Technical Memorandum 103805

\title{
IMPAC - An Integrated Methodology for Propulsion and Airframe Control
}

Sanjay Garg, Peter J. Ouzts, and Carl F. Lorenzo

National Aeronautics and Space Administration

Lewis Research Center

Cleveland, Ohio

and

Duane L. Mattern

Sverdrup Technology, Inc.

Lewis Research Center Group

Brook Park, Ohio

Prepared for the

American Control Conference

sponsored by the American Automatic Control Council

Boston, Massachusetts, June 26-28, 1991 


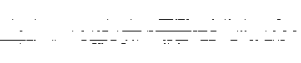
$+2$

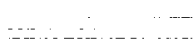

(

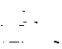

$-$

$-\mathrm{X}$ 


\title{
IMPAC - an Integrated Methodology for Propulsion and Airframe Control
}

\author{
Sanjay Garg, Peter J. Ouzts, Carl F. Lorenzo and Duane L. Mattern1 \\ NASA Lewis Research Center \\ Advanced Control Technology Branch \\ Cleveland, Ohio 44135
}

\begin{abstract}
NASA is actively involved in the development of enabling technologies that will lead towards aircraft with new/enhanced maneuver capabilities such as Short Take-Off Vertical Landing (STOVL) and high angle of attack performance. Because of the high degree of dynamic coupling between the airframe and propulsion systems of these types of aircraft, one key technology is the integration of the flight and propulsion control. The NASA Lewis Research Center approach to developing Integrated Flight Propulsion Control (IFPC) technologies is an in-house research program referred to as IMPAC - Integrated Methodology for Propulsion and Airframe Control. The goals of IMPAC are to develop a viable alternative to the existing integrated control design methodologies that will allow for improved system performance and simplicity of control law synthesis and implementation, and to demonstrate the applicability of the methodology to a supersonic STOVL fighter aircraft. Based on some preliminary control design studies that included evaluation of the existing methodologies, the IFPC design methodology that is emerging at the Lewis Research Center consists of considering the airframe and propulsion systems as one integrated system for an initial centralized controller design and then partitioning the centralized controller into separate airframe and propulsion system subcontrollers to ease implementation and to set meaningful design requirements for detailed subsystem control design and evaluation. This paper provides an overview of IMPAC and includes detailed discussion of the various important design and evaluation steps in the methodology.
\end{abstract}

DDr. Mattern is with Sverdrup Technology Inc. 


\section{Introduction}

The desire to enhance the overall performance of complex, multi-element, dynamically coupled systems has fostered much research in integrated sys tem control design methodologies. Significant performance gains may be achievable by forjnulating the multi-element, dynamically coupled system control design problem in an integrated system framework rather than an after-the-fact, ad hoc combination of individual element control designs. The goal of integrated controls research is to develop integrated control design methodologies, and demonstrate their advantages, such that these methodologies are available to the control system designer when faced with the multi-element, dynamically coupled system control design task.

One example of a multi-element, dynamically coupled system which has been the subject of control design methodology research is the Short TakeOff and Vertical Landing (STOVL) aircraft. The use of forces and moments produced by the propulsion system to extend the flight envelope of the aircraft results in significant dynamical coupling between the airframe and propulsion systems. This coupling is such that traditional methods of aircraft control design, (e.g., separately designed flight control and propulsion control systems) are not adequate to the task. While a STOVL aircraft and its accompanying flight and propulsion control systems have been operating successfully for some years (i.e., the AV8-Harrier), the STOVL aircraft concepts currently under study include supersonic flight capabilities and therefore embody significantly more complex systems than previous subsonic STOVL configurations. The additional system complexity present in the supersonic STOVL aircraft and the significant potential performance gains in terms of pilot workload reduction and improved dynamic performance that may be realized from an integrated control design approach have spurred research into Integrated Flight and Propulsion Control (IFPC). The goal of the IFPC research has been the development and validation of control design methodologies which directly consider the significant dynamical coupling between the airframe and propulsion systems of STOVL aircraft and thereby 
result in improved overall system performance. Further, while integration of the flight and propulsion controls is explicitly addressed, the effort attempts the control integration of more than two subsystems towards the more generic control integration problem.

In the early 1980's, the Air Force commissioned a study called Design Methods for Integrated Control Systems (DMICS). This study sought to identify and develop IFPC control laws for an advanced tactical aircraft. The particular aircraft configuration chosen for the DMICS study included thrust-vectoring and thrust-reversing capabilities which resulted in additional dynamic coupling (beyond a conventional aircraft configuration) between the propulsion and airframe systems. The results of the study were two IFPC design methodologies, 1) a centralized design approach ,Ref. [1], and 2) a decentralized, hierarchical approach, Ref. [2], to be applied to the IFPC design problem. Subsequent to the Air Force DMICS studies, the NASA Lewis Research Center (LeRC) identified IFPC as an enabling technology for supersonic STOVL aircraft and initiated research in this area. One study, under a NASA Lewis and Ames sponsored program contracted to an industry team, seeks to apply the DMICS decentralized, hierarchical design approach to an ejector configured supersonic STOVL aircraft (E-7D), Ref. [3]. The goal of this study is to determine the applicability of DMICS decentralized, hierarchical design approach to the supersonic STOVL IFPC design problem. In parallel to this sponsored program, NASA LeRC began an in-house study of IFPC design methodologies. The goal of the NASA LeRC in-house research program is to further study existing IFPC design methodologies to identify their strengths/weaknesses and, based on this experience, make modifications to the existing IFPC methodologies and/or formulate new integrated control design methodologies that can be applied to a broad class of aerospace systems that exhibit strong coupling between the various sub-systems.

The NASA LeRC in-house research program has been named Integrated Methodology for Propulsion and Airframe Control (IMPAC). The first phase of the IMPAC program was evaluation studies of the two DMICS control design methodologies. 
These studies sought to further identify positive and negative aspects of the DMICS design methodologies to allow a judgment of their applicability to IFPC design. The centralized DMICS study, Ref. [4], showed that while the centralized approach promises "optimal" system performance with respect to the specified design criteria, it suffers from control design technique limitations which result in a complex centralized controller. Difficulties would arise in implementing a centralized controller in existing airframe-propulsion control system structures and there are issues of independent subsystem (e.g., propulsion system) control validation. The decentralized, hierarchical studies, Ref. [5], showed that the decentralized approach resulted in simpler, easy to implement controllers and allowed the individual subsystem control designers to formulate their control designs to best match the subsystem performance requirements. However, all the various subsystem interactions cannot be accounted for within an integrated framework in the decentralized approach. So this approach is fundamentally highly iterative and would generally yield inferior performance due to its inherent design conservatism. Also, the assumption of weakly coupled subsystems is critical to the successful application of the decentralized approach. Thus a decentralized control design approach might not meet the integrated control requirements for a system consisting of highly coupled subsystems.

Based on the experience gained with the example DMICS design studies, the IMPAC program has formulated a new IFPC design methodology that considers the airframe and propulsion systems as one integrated system. An initial centralized controller design for the integrated system will be performed at each design point using appropriate modern multivariable robust control design techniques. Note that in this context, centralized controller implies a controller designed using a plant model obtained by integrating all the subsystems, and such that the controller acts on all the outputs of the integrated plant to generate commands for all the control inputs of the integrated plant without any pre-specified controller structure. Sometimes the word "global" is used in the control literature to describe this type of controller. These centralized controllers will then 
be partitioned into separate airframe and propulsion system subcontrollers to ease implementation, address nonlinear issues and allow independent subsystem validation. The vehicle to be used for development and validation of the IMPAC design methodology is the same ejector configured E-7D aircraft being used in the industry IFPC study. Since the centralized control design under IMPAC will be done using modern multivariable control synthesis techniques, an important research goal for the IMPAC program will be to determine whether these techniques can be effectively used to design IFPC systems for operation over the entire flight envelope of a supersonic STOVL aircraft.

In the following, the overall IMPAC design methodology is first presented and the various elements of the methodology are briefly described. This is followed by a more detailed discussion of those individual areas which are critical for overall IFPC methodology development and demonstration, and/or areas in which the IMPAC design approach differs significantly from the existing IFPC design techniques. The paper concludes with a brief summary of the goals of the IMPAC program and the achievements to date.

\section{IMPAC - Methodology Philosophy}

The philosophy driving the formulation of the IMPAC design methodology is that maximum performance (e.g., pilot handling qualities, pilot workload, etc.) from an integrated system will best be achieved when a highly integrated control design process is used. Accordingly, the IMPAC design methodology strives to provide the control designer with a well-defined, integrated control design approach to the IFPC problem. The IMPAC design approach to the IFPC control problem is a centralized control design for the integrated airframe/propulsion system followed by partitioning of the centralized linear controller into separate airframe and propulsion linear controllers. The centralized linear control design approach best considers the integrated nature of the control design problem. However, a centralized controller may be neither consistent with current IFPC implementations nor best suited to the individual elements (e.g., propulsion system) 
resulting from this implementation. Partitioning of the centralized controller into subsystem controllers establishes a performance baseline to be met during subsystem control design, allows for the realistic implementation of the centralized control laws, and also permits the inclusion of specific nonlinear control logic pertinent to a particular subsystem (for example, propulsion system safety limits). Therefore, included in the IMPAC design approach is the mathematical basis to perform this centralized controller partitioning.

An overall system control design process consists of many distinct tasks, not all of which are strictly control design tasks. Some of these tasks are common to any control design methodology (e.g., definition of the system requirements, system dynamic modeling) and therefore are not specifically addressed by the IMPAC design methodology. Figure 1 shows the relationship of IMPAC in an overall system control design process. The IMPAC design process requires several forms of information about the system to begin the IFPC design process. The aircraft and propulsion system configuration and requirements must be defined. Desired performance (e.g., handling qualities) must be formulated. Full nonlinear dynamic models of the airframe and propulsion system are needed. Finally, the necessary open-loop dynamic studies of system performance need to be performed. With this information the IMPAC design methodology formulates the control laws for the IFPC system. These control laws are then subjected to a variety of evaluations including pilot-in-the-loop testing. Results of these evaluations are used in subsequent control law design iterations.

\section{IMPAC - Methodology Flowchart}

A flowchart of the IMPAC design methodology is presented in Fig 2. This flowchart identifies major tasks and features of the IMPAC approach and provides a detailed view of the methodology. What follows here is a brief description of the design steps as shown in Fig. 2. A more detailed technical description of the tasks central to the IMPAC methodology are presented later in this paper. 
Given that integrated, nonlinear dynamic models for the system are available, the first task in the IMPAC design methodology involves generation of dynamic models to be used for control law synthesis (Block 1). These control design models are, in general, traditional linear perturbation models of the system taken at various operating points. It should be emphasized that the linear models used for control design are of the integrated system, i.e., airframe and propulsion systems along with coupling dynamics. An important issue in a centralized linear IFPC design approach is how nonlinearities of subsystems (e.g., propulsion system) will effect the validity of the centralized linear control law synthesis. Specifically, propulsion system nonlinearities such as fuel flow acceleration/deceleration rates will not be reflected in small perturbation linear models and therefore cannot enter the centralized control design process. However, in final implementation, these nonlinearities may in fact dominate the subsystem response.

One method to address the issue of subsystem nonlinearities being investigated by IMPAC is how nonlinearities can be considered before the linear control law design process begins. Therefore, some "conditioning" of the control design models is envisioned in the control model generation task of the IMPAC approach. This conditioning may include such features as feedback linearization or large perturbation linearization such that the effects of subsystem nonlinear behavior enter early in the control design process. The final product of the control model generation process is a set of operating point specific, linear, state-space dynamic models of the integrated system that will allow a "realistic" centralized control design.

The centralized control law design process and subsequent controller partitioning are major elements of the IMPAC methodology. The centralized control design process (Block 2) uses the full system state-space linear control design models previously developed and is based on available multivariable linear control design techriques that have the capability to meet the IFPC requirements. Design criteria formulated from system performance requirements and system open-loop dynamic studies provide the 
necessary control design specifications (e.g., frequency or time dependent weighting factors) for the chosen linear design technique. The centralized control law is evaluated against the design specifications and appropriate modifications made. Because the linear control law synthesis tool may result in a high order centralized controller, controller reduction may be performed at this point in the method. A detailed description of centralized control law design is presented later in the paper. The result of this process is an operating point specific, centralized linear feedback controller for the integrated system.

Once the centralized controller is designed, mathematical techniques have been developed to partition the centralized linear controller into linear sub-controllers (Block 3). The controller partitioning task requires that a candidate control structure for the partitioned system be specified. For example, for the IFPC problem, the assumed control structure is hierarchical with the airframe (flight) control partition exercising some authority over the propulsion partition. The IMPAC methodology allows many candidate control structures to be considered at the partitioning level based upon the requirements of the system. Comparisons between the centralized and partitioned linear controllers are made to validate the partitioning process. Subsystem controller reduction may take place as desired. Again, a detailed description of the controller partitioning process is presented later in the paper. The result of the controller partitioning task is a set of subsystem linear controllers which, when reassembled, approach (within some desired tolerance) the performance of the centralized control design.

After completion of the linear control design tasks, individual subsystem control design must be performed. In general, the subsystem control design task will include accounting for the effects of subsystem nonlinearities in the subcontrollers resulting from the controller partitioning task. Several types of subsystem nonlinearities are considered in IMPAC. The first involves extension of the individual subsystem controllers to full envelope (as defined by the system requirements) operation (Block 4). Typically this would involve gain scheduling of individual operating point controllers to account for 
parameter variations (e.g., control power, atmospheric conditions). It is envisioned that the use of modern robust control synthesis tools will ease the burden of the full envelope control formulation.

The second subsystem nonlinear control design task (Block 5) incorporates any additional subsystem nonlinearities into the subsystem controllers. Nonlinearities such as propulsion system safety limits are considered in this task. For example, the propulsion system would require fuel flow control limit logic to ensure that engine surge margins are maintained. After the appropriate nonlinear control loops have been designed, the subcontrollers can be validated using the appropriate subsystem dynamic models. A more detailed discussion of subsystem nonlinearities considered in IMPAC is presented later in the paper. The result of this task is the nonlinear limit and accommodation logic to be added to the full envelope subsystem controllers.

The final task in the IMPAC design approach is reassembly of the full envelope, nonlinear subsystem controllers to form the desired implementation of the centralized control law (Block 6). Additional design options such as global nonlinear optimization may be performed at this point. Evaluations of the final controller would take place using both nonlinear dynamic models and pilot-in-the-loop (PITL) facilities. These evaluations would test the actual system performance (e.g., handling qualities) against the desired system performance specifications.

It should be noted that as with any design process, actual implementation of the IMPAC methodology will likely involve iterative design steps. For example, the nonlinear design evaluation may expose certain design deficiencies which require reformulation of centralized linear control laws. However, the integrated control design approach contained in the IMPAC methodology will reduce the number and severity of the design iterations.

Integrated Control Design

The IMPAC control design structure is as shown in Fig. 3. The control elements to be designed are the Configuration Management, Command Shaping/Mode Switching Logic, 
the Integrated Control consisting of the Airframe and Propulsion system subcontrollers, and Sensor Compensation. The Configuration Management consists of generating trim command information for the small perturbation command tracking inner-loop integrated control based on the pilot commands and the aircraft mode of operation. The Command Shaping/Mode Switching logic consists of converting pilot inputs to the control effectors into commands to be tracked by the integrated control and its design is driven by the handling qualities requirement. This logic also ensures a smooth transition between aircraft operational control modes. The Sensor Compensation design consists of generating the inputs to the integrated control from the various aircraft measurements and will include things like sensor aliasing and normalization, estimation using complimentary filters etc. The design of these elements other than the Command Shaping portion, i.e. Configuration Management, Mode Switching logic and Sensor Compensation, under IMPAC will be done using standard techniques such as those used in in the ongoing STOVL Controls contract program (Ref. [6]), for example. So these elements are not discussed further in this paper. The major development effort under IMPAC is on the Integrated Control design which consists of linear control design followed by nonlinear extension of the controller for operation over the flight envelope of the aircraft.

As shown in Fig. 4, the linear control design portion of the IMPAC approach consists of three major design steps: (i) Design of a centralized feedback controller to provide command tracking, stability and performance robustness, based on the fully integrated airframe/propulsion model as a single high-order system, (ii) Partitioning of the centralized controller into decentralized subcontrollers compatible with desired structure of control implementation, and (iii) Design of command shaping prefilters from pilot control effectors to commanded variables to provide the overall desired response to pilot inputs. The command shaping prefilter design will be accomplished using the multivariable band-limited inverse method of Ref. [7]. This method consists of formulating the prefilter synthesis problem within the framework of the linear quadratic stochastic control problem 
with the feedback closed-loop system as the plant. This formulation provides for setting the bandwidth of the prefiltering action by properly selecting the control weighting in a regulator problem. Such an approach allows the control designer to obtain a prefilter for desired response to command inputs without altering the feedback properties of the control loop. This approach has been successfully demonstrated in Refs. $[1,4]$ and is not further discussed further in this paper. The details of centralized controller design, controller partitioning and nonlinear control extensions are discussed in the following.

\section{Centralized Controller Design}

The major issue related to the feedback controller design portion of IMPAC, i.e. Block 2 of Fig. 2, is the choice of the control synthesis technique that "best" suits the IFPC objectives. Not only should the synthesis technique allow a formulation of centralized control design criteria that adequately reflects the performance specifications of the "total" system i.e. the airframe integrated with the propulsion system, but it should also result in controllers of reasonable order with guaranteed performance and robustness characteristics. Robustness is of special importance because there are many modelling uncertainties and errors associated with the design plant due to neglected/unknown dynamics, nonlinearities, parameter uncertainties, actuator rate and limit saturations, etc. The Linear Quadratic Gaussian/Loop Transfer Recovery (LQG/LTR) approach was used for the centralized IFPC control design in the DMICS study of Ref. [1]. However, as pointed out in Ref.[4], the strength of the LQG/LTR methodology is synthesizing command tracking control laws. One of its weaknesses is its inability to address the issue of plant augmentation. Since plant augmentation is an integral part of flight control law design, and because of other drawbacks associated with the LQG/LTR technique (see discussion in Refs. $[4,8]$ ), this methodology was considered to be inadequate for the task of centralized IFPC design. Other multivariable control design techniques such as LQG, optimal control using parameter optimization, multivariable root-loci, etc. do not provide any guaranteed robustness characteristics and/or are in general too cumbersome to apply to large, strongly 
coupled systems in that these are too computationally intense requiring many iterations over the design parameters.

An emerging control synthesis technique that has the promise to meet the IFPC design requirements is the $\mathrm{H}$-infinity $\left(\mathrm{H}_{\omega}\right)$ control theory (Refs. $\left.[9,10]\right)$. Recent advances in computational algorithms (Ref. [11]) have made this theory a viable candidate to be applied to complex multivariable control design problems. In gross terms, this technique provides the designer the means to synthesize a controller for "best" guaranteed performance in the presence of "worst case" disturbances (and/or commands). Proper formulation of the control design problem using $H_{\infty}$ theory provides for building-in stability robustness and obtaining an adequate trade-off between performance and allowable control power in the resulting controller. Recent preliminary studies of IFPC design for STOVL aircraft using $H_{\infty}$ control theory have been very successful (Refs. $[12,13])$, thus affirming the viability of using this approach for centralized feedback control design portion of IMPAC. It is important to note here that the overall integrated control design philosophy under IMPAC is not dependent on any particular choice of control synthesis technique. Any future synthesis technique which has the capability to meet the IFPC design requirements stated earlier can be substituted in place of the current choice of $\mathrm{H}_{\mathrm{w}}$ control synthesis.

The detailed block diagram for the $\mathrm{H}_{\infty}$ formulation of the IFPC feedback control design within the framework of a command tracking problem is shown in Fig. 5. The three transfer functions that are of interest for such a problem are the sensitivity function $S(s)$, the complementary sensitivity function $T(\mathrm{~s})$, and the control transmission function $\mathrm{C}(\mathrm{s})$. These represent the closed-loop transfers from the reference commands (and disturbances) to tracking errors, controlled variables and commanded control inputs, respectively, i.e. $\mathrm{e}=\mathrm{S}(\mathrm{s}) \mathrm{z}_{\mathrm{c}}, \mathrm{z}=\mathrm{T}(\mathrm{s}) \mathrm{z}_{\mathrm{c}}$ and $\mathrm{u}=\mathrm{C}(\mathrm{s}) \mathrm{z}_{\mathrm{c}}$. In order to influence both the low-frequency and high-frequency properties of the closed-loop system it is desirable to find a controller $K(\mathrm{~s})$ which minimizes a weighted norm of a combination of these three transfer functions, i.e. 


$$
\min \|H(j \omega)\|_{\infty} \text { with } H(j \omega)=\left[\begin{array}{l}
W_{S}(j \omega) \cdot S(j \omega) \\
W_{T}^{(j \omega)} \cdot T(j \omega) \\
W_{C}(j \omega) \cdot C(j \omega)
\end{array}\right]
$$

Note that $\|\mathrm{H}(\mathrm{j} \omega)\|_{\infty}$ is the highest value over all frequencies $\omega$ of the maximum singular value of $\mathrm{H}(\mathrm{j} \omega)$, i.e. $\|\mathrm{H}(\mathrm{j} \omega)\|_{\infty}=\max _{\omega} \bar{\sigma}[\mathrm{H}(\mathrm{j} \omega)]$.

The weighting functions $\mathrm{W}_{\mathrm{S}}(\mathrm{j} \omega), \mathrm{W}_{\mathrm{T}}(\mathrm{j} \omega)$, and $\mathrm{W}_{\mathrm{C}}(\mathrm{j} \omega)$ are the parameters used by the control designer to "tune" the controller $\mathrm{K}(\mathrm{s})$ such that the design objectives are met. For instance choosing $\mathrm{W}_{\mathrm{S}}$ to be large at low frequency ensures good command tracking performance and choosing $\mathrm{W}_{\mathrm{T}}$ to be large at high frequencies ensures robustness to high frequency unmodelled dynamics. The control weighting $W_{C}$ is chosen to ensure that control actuation bandwidths, rate and position requirements are practically achievable.

A procedure for building-in robustness to plant modeling uncertainties within the framework of the $\mathrm{H}_{w}$ control problem formulation is to use fictitious internal model disturbances as exogenous inputs to the design plant. The disturbances models are developed to mimic the effects of modelling uncertainties. These uncertainties are quantified during the design model development work. Such a formulation has been shown to induce robustness in the $\mathrm{H}_{\infty}$ control design in Ref. [14], and has been successfully applied to robust IFPC design in Ref. [13].

\section{Controller Partitioning}

Generally, the centralized IFPC controller obtained by application of the above procedure will be of high order and generally too complex to implement. Such a controller might also contain many feedback paths which are not physically realizable. Also, traditionally it is the responsibility of the engine designer/manufacturer to ensure that the engine will provide the desired performance when installed in the airframe. The engine manufacturer also needs a separate engine controller to be able to independently perform extensive testing to assure an adequate design and engine integrity. To address these difficulties, the idea of partitioning the çentralized controller (Block 3 in Fig. 2) into 
separate airframe and propulsion system subcontrollers was introduced in Ref. [4]. The desired structure of controller partitioning will depend on the coupling between the various subsystems and on practical considerations related to integration of the independently controlled subsystems. A decentralized, hierarchical control structure as shown in Fig. 6 was chosen for controller partitioning in IMPAC. In Fig. 6, the subscript "a" refers to airframe quantities, "e" refers to propulsion system quantities, and "c" refers to commands. The intermediate variables, $z_{\text {ea, }}$ represent propulsion system quantities that affect the airframe, for example propulsive forces and moments. The partitioning shown in Fig. 6 is simplified in that the controlled output errors are assumed to be the only inputs to the subcontrollers, i.e. the measurement vector y of Fig. 4 is assumed to be zero. This assumption is being made here strictly to keep the following discussion simple while conveying the basic idea behind formulating and solving the controller partitioning problem. The actual IMPAC proof-of-concept designs will be done using both $\mathrm{y}$ and $\mathrm{z}$. The controller partitioning problem of Fig. 6 can be stated as follows :

Given: $\quad K(s)$ s.t. $u(s)=K(s) \cdot e(s)$, where $u=\left[\begin{array}{l}u_{a} \\ u_{e}\end{array}\right]$ and $e=\left[\begin{array}{l}e_{a} \\ e_{e}\end{array}\right]$,

Find: $\quad \mathrm{K}_{\mathrm{a}}(\mathrm{s}), \mathrm{K}_{\mathrm{e}}(\mathrm{s})$ and a particular set of $\mathrm{z}_{\mathrm{ea}}$ with

$$
\left[\begin{array}{r}
u_{a}(s) \\
z_{e a}(s)
\end{array}\right]=K_{a}(s) \cdot e(s) \text { and } u_{e}(s)=K_{e}(s) \cdot\left[\begin{array}{r}
e_{e}(s) \\
e_{e a}(s)
\end{array}\right]
$$

So that: The closed-loop performance and robustness with the subcontrollers $\mathrm{K}_{\mathrm{a}}(\mathrm{s})$ and $\mathrm{K}_{\mathrm{e}}(\mathrm{s})$ match those with the centralized controller $\mathrm{K}(\mathrm{s})$ to a desired accuracy.

A state-space parameter optimization based algorithmic procedure to solve the above controller partitioning problem is currently being developed. Some preliminary results using this approach are available in Ref. [15]. The major steps in this approach to controller partitioning are shown in the flow chart of Fig. 7 and are discussed in the following. 
Step 1: $\quad$ Make an initial guess for the state-space parameters of the airframe and propulsion subcontrollers. In order to keep the number of parameters to the smallest possible value for the specified subcontroller orders, the structure of the state-space matrices of the subcontrollers will be chosen as suggested in Ref. [16]. Since numerical parameter optimization techniques are very sensitive to the initial guess for the parameters, it is very important to start the iterations with a "good" initial guess. A systematic procedure for determining an initial controller partitioning is documented in Ref. [17]. The application of this procedure to an example IFPC design in Ref. [17] resulted in good matching of the centralized controller performance with the initial partitioned subcontrollers thus demonstrating the feasibility of the controller partitioning idea.

Step 2: Calculate the state-space representation of the controller $(\overline{\mathrm{K}}(\mathrm{s}))$ obtained by "assembling" the partitioned subcontrollers. This "assembly" is done by using the plant information to remove the controller dependence on the interface variables $z_{e a}$ so that the assembled controller will have the same inputs and outputs as the original centralized controller. The cost to be minimized in the optimization procedure is chosen to be the infinity norm of the weighted difference between the centralized controller and the assembled controller, i.e., Minimize $J(P)=\|(K-\bar{K}(P)) \cdot W(j \omega)\|_{\infty}$ where $P$ is the set of design parameters for optimization. Minimizing the infinity-norm ensures that the assembled controller closely approximates the centralized controller, and the frequency dependent weighting is used to emphasize the frequency range and the directions in which a "good" approximation is desired. For instance, choosing $W(j \omega)=G(j \omega)$, the controlled plant, ensures that the closed loop system with the partitioned controllers will closely match the performance and robustness characteristics of the system with the centralized controller.

Step 3: Check whether the cost for the parameter set at step $i, J\left(P_{i}\right)$, is the minimum achievable. If yes, then go to Step 6, otherwise go to Step 4 .

Step 4: Calculate the gradient of the cost with respect to the parameters $P_{i}$. To 
speed up the computations, analytical expressions for the gradients will be used in the partitioning computer codes.

Step 5: Calculate the next feasible set of parameters which lowers the cost. Standard algorithms such as the Quasi-Newton method with BFGS (Broyden-Fletcher-Goldfarb-Shano) update of the Hessian approximation [18] will be used to implement this step. Go to Step 2.

Step 6: Once the "optimal" set of parameters is obtained, the closed-loop system performance and robustness with the partitioned controllers is evaluated and compared with that achieved with the centralized controller. If the performance achieved with the subcontrollers is not acceptable, then the partitioning procedure is repeated with one or more of the following changes : (i) Different initial guess for the parameters keeping the subcontroller orders the same, (ii) Increased order of airframe and/or propulsion subcontrollers, (iii) Different choice of weighting $\mathrm{W}(\mathrm{j} \omega)$, and finally (iv) Different partitioning structure i.e. changing the split of the centralized controller inputs and outputs between the subcontrollers (choice of $e_{a}, e_{e}$ and $u_{a}, u_{e}$ respectively), and/or different choice of the interface variables $z_{e a}$.

Once an acceptable controller partitioning is obtained, the subcontrollers may be further reduced in order using modern controller reduction techniques and will be simplified by removing the feedback paths of least significance. Note that by comparing the performance obtained by various partitioned controller implementations of different level of complexities with that obtained by the centralized controller, a meaningful trade-off can be obtained between implementation controller complexity and achievable performance.

\section{Nonlinear Considerations}

Nonlinearities that should be accounted for in the IFPC design can be grouped as follows: variations in the plant system matrices $(A, B, C \& D)$ caused by changes in the nominal operating condition; "hard" actuator limits; and operational limits imposed on the plant outputs. It is assumed that variations in the matrix elements of a linear model are 
slow relative to the plant dynamics (Ref. [19]). For example, changes in the lift coefficient as a function of dynamic pressure appear as variations in the matrices of an airframe model. "Hard" actuator limits consist of mechanical stops and slewing rates imposed by the hardware. The maximum rate of change of an engine nozzle area and the maximum flap deflection are examples of "hard" actuator limits. Safety limits are self imposed limits on the plant output required to protect and to extend the life of the plant. If the limited output is unmeasurable, then the limit can be reflected back to the inputs based on a model of the plant. Safety limits are typical in a turbojet engine and a fuel flow acceleration/deceleration schedule is an example of such a limit. The acceleration/deceleration schedule bounds the engine fuel flow to limit the maximum turbine temperature and the minimum compressor surge margin (Ref. [20]). An example of a measurable safety limit in a turbojet engine is the limit placed on the maximum fan rotor speed.

It is possible to address certain nonlinearities in the linear control design. Slow variations in the plant system matrix ("A"), can be modelled as uncertainties and can be addressed using robust control design techniques. Gross variations in the control effectiveness matrix (" $\mathrm{B}$ ") are best addressed outside of the linear control design using gain scheduling (Ref. [21,22]). Actuator rate and range limits can be accounted for in the linear control design. Consider a rudder actuator with a mechanical range of $+1-20$ degrees and a maximum rate of change of 40 degrees/sec. These range and rate limits can be built into a linear design structure as inverse weights on the control and control rate authority. Weighing both the range and the rate constitutes a frequency weight for the actuator. The use of these weights does not guarantee that the limits will not be exceeded by the linear controller, only that the linear control design will provide an appropriate performance/control trade-off and will distribute the control action among the actuators relative to their control effectiveness. These actuator weights direct the design method to "optimally" use the available control authority to achieve the desired specifications. Safety 
limits can also be considered in the linear design. For example, in a turbofan engine the difference between the fuel flow for the nominal operating condition and the upper and lower fuel flow limits due to the acceleration/deceleration schedule can be used to select a scale value for the fuel flow actuator in the linear design at that operating point. This scale factor allows the control system designer to weigh the consequences of exceeding the fuel flow limit versus using the other actuators to achieve the desired specifications.

Nonlinearities that cannot be addressed in the linear design need to be addressed by other methods. Figure 8 shows the major components of a typical nonlinear subcontroller. Gain scheduling is used to account for variations in control effectiveness due to changes in the nominal operating condition. For example, in the STOVL IFPC problem, during transition from cruise to hover, as the aircraft slows down, propulsive lift replaces aerodynamic lift as a means to support the weight of the aircraft. As the velocity of the aircraft decreases, the engine power is increased to provide the necessary lift. Increasing the engine power increases the mass flow through the nozzles which changes the control effectiveness of the nozzles. Also, the control effectiveness of the aerodynamics surfaces are reduced due to the reduction in dynamic pressure. The gain scheduler shown in Fig. 8 accounts for gain change and the control power redistribution. Since zero steady state error is a typical control objective and is achieved using integrators, integrator windup protection is required whenever the actuator are limited. There are a variety of integral windup protection schemes (Refs. [23,24]). Figure 8 shows one possible structure for a windup protection scheme (Ref. [25]) that modifies the errors that drive the linear controller. The mode selection logic indicated in Fig. 8 selects the errors used in the control which are dependent on the limit operation and is typical of turbofan engine operation (Refs. $[26,27]$ ).

In IMPAC, the partitioning of the linear controller results in a hierarchical structure. A limit encountered in a lower level subsystem has to be communicated back to a higher level if it affects the overall performance. This requires a communication path 
from the engine limit logic back to the higher level airframe control redistribution/gain scheduling logic to communicate when the engine has encountered a limit that will affect the thrust response, as suggested in reference Ref. [28]. If the desired performance can not be maintained by redistributing the control authority, then the airframe controller logic must decide how to distribute the performance degradation. For example, if additional thrust is requested from one of three engine nozzles on the E-7D aircraft, and the rate of change of gross thrust is limited by the fuel flow acceleration schedule, then the resulting thrust error can be lumped into one nozzle if the response from that nozzle is noncritical. Alternatively, the thrust error can be equally distributed over all nozzles. This decision is made at the higher level subcontroller and requires the limit information of all lower level subcontrollers.

These are concepts that will be used under IMPAC to extend the linear IFPC design to full-envelope nonlinear control. Note that the controller partitioning discussed earlier will ease this task because it allows for treating the airframe and propulsion system nonlinearities separately and for each subsystem the critical safety and limit protection logic can be "wrapped" around the corresponding subcontroller.

\section{Summary}

A methodology called IMPAC (Integrated Methodology for Propulsion and Airframe Control) for integrated flight/propulsion control design for future aircraft with enhanced maneuver capabilities was discussed. The significant features of this methodology are that it consists of first designing a centralized controller considering the airframe and propulsion systems as one integrated system and then partitioning the centralized controller into decentralized subcontrollers (sub-system controllers) with a specified interconnection structure. The centralized control design accounts for all the subsystem interactions at the design stage and provides a baseline for the "best" achievable performance with a fully integrated system. The partitioning results in easy to implement subcontrollers that allow for independent detailed subsystem control design and validation. 
The controller partitioning also allows for the system nonlinearities to be considered in detail at the subsystem level. A meaningful trade-off between subcontroller complexity and achievable performance for the integrated system can be performed by evaluating various controller partitionings of different levels of complexities against the performance baseline established with the centralized controller. It is expected that this methodology will lead to control designs which provide improved system performance over the existing integrated control design methodologies and are easy to implement. The IMPAC methodology is to be demonstrated by application to a currently envisioned Short Take-Off and Vertical Landing (STOVL) fighter aircraft which is powered by a high bypass turbofan engine and is equipped with ejectors to provide propulsive lift at low speeds and hover.

To date, an emerging multivariable control synthesis technique that meets the requirements of the centralized control design portion of IMPAC has been identified and the preliminary results obtained with this technique are encouraging. A decentralized, hierarchical controller partitioning structure which yields a practically implementable integrated flight propulsion control design has been identified and the theoretical formulation for such a controller partitioning has been completed. Numerical algorithms to implement the controller partitioning procedure are currently being developed. Development of various control design and evaluation models and integrated simulations for the study STOVL aircraft is complete and the control design using IMPAC is progressing. Preliminary piloted simulation evaluation of the IMPAC based integrated flight propulsion control design using the NASA Lewis Research Center fixed-base simulator is planned for the end of the year. 


\section{References}

[1] Smith, K.L., "Design Methods for Integrated Control Systems," AFWAL-TR-86-2103, Wright Patterson AFB, Dayton, OH, December 1986.

[2] Shaw, P.D., "Design Methods for Integrated Control Systems," AFWAL-TR-88-2061, Wright Patterson AFB, Dayton, OH, June 1988.

[3] Mihaloew, J.R., and Drummond, C.K., "STOVL Aircraft Simulation for Integrated Flight and Propulsion Controls Research," NASA TM-102419, June 1989.

[4] Garg, S., Mattern, D.L., and Bullard, R.E., "Integrated Flight/Propulsion Control System Design Based on a Centralized Approach," Journal of Guidance, Control and Dynamics, Vol. 14, No. 1, Jan.-Feb. 1991, pp. 107-116.

[5] Mattern, D.L., Garg, S., and Bullard, R.E., "Integrated Flight/Propulsion Control Design Based on a Decentralized Approach," AIAA Paper p 89-3519, Guidance Navigation and Control Conference, Boston, MA, August 1989

[6] "E-7D Flight Control System Description," Systems Control Technology, Palo Alto, CA, May 1990. Report prepared for GE Aircraft Engines under Contract No. 200-14-14P94337.

[7] Lehtomaki, N.A., Stein, G., and Wall, J.E., "Multivariable Prefilter Design for Command Shaping," AIAA Paper 84-1829, Guidance, Navigation and Control Conference, Seattle, WA, August 1984.

[8] Stein, G., and Doyle, J.C., "Beyond Singular Values and Loop Shapes," Journal of Guidance, Control and Dynamics, Vol. 14, No. 1, Jan.-Feb. 1991, pp. 5-16.

[9] Doyle, J.C., Glover, K., Khargonekar, P.P., and Francis, B.A., "State-Space Solutions to Standard $\mathrm{H}_{2}$ and $\mathrm{H}_{\infty}$ Control Problems," IEEE Transactions on Automatic Control, Vol. 34, No. 8, August 1989, pp. 831-847.

[10] Safonov, M.G., Limebeer, D.J.N., and Chiang, R.Y., "Simplifying the $\mathrm{H}$ Theory via Loop-Shifting, Matrix Pencil and Descriptor Concepts," Int. J. of Control, 1989, Vol. 50, No. 6, pp. 2467-2488.

[11] "MATRIXx Robust Control Module," Integrated Systems Inc., Santa Clara, CA, December 1989.

[12] Garg, S., Mattern, D.L., Bright, M.M., and Ouzts, P.J., "H-Infinity Based Integrated Flight/Propulsion Control Design for a STOVL Aircraft in Transition Flight," AIAA Paper 90-3335, Guidance, Navigation and Control Conference, Portland, OR, August 1990.

[13] Garg, S., and Ouzts, P.J., "Integrated Flight/Propulsion Control Design for a STOVL Aircraft Using H-Infinity Control Design Techniques," 1991 American Control Conference, Boston, MA, June 1991.

[14] Reichert, R., "Application of H-Infinity Control to Missile Autopilot Design," 
AIAA Paper 89-3550, Guidance, Navigation and Control Conference, Boston, MA, August 1989, pp. 1065-1072.

[15] Schmidt, P., and Garg, S., "Decentralized Hierarchical Partitioning of Centralized Integrated Controllers," 1991 American Control Conference, Boston, MA, June 1991.

[16] Ly, U.L., Bryson, A.E., and Cannon, R.H., "Design of Low-Order Compensators using Parameter Optimization," Automatics, Vol. 21, No. 3, pp. 315-318, 1985.

[17] Garg, S., "Controller Partitioning for Integrated Flight/Propulsion Control Implementation," NASA TM under preparation, NASA Lewis Research Center, Cleveland, $\mathrm{OH}$.

[18] Dennis, J.E., and More, J.J., "Quasi-Newton Methods, Motivation and Theory," SIAM Review, 19, 1977, pp. 46-89.

[19] Shamma J.S., Athans M., "Analysis of Gain Scheduled Control for Nonlinear Plants", IEEE Automatic Control, August 1990, Vol 35, No. 8, p 898.

[20] Sobey A.J., Suggs A.M., Control of Aircraft and Missile Powerplants, John Wiley and Sons, Inc., New York, N.Y., 1963, p 185.

[21] Kapasouris P., Athans M., and Spang H.,A. III, "Gain- Scheduled Multivariable Control for the GE-21 Turbofan Engine using the LQG/LTR Methodology", 1985 American Control Conference, p109.

[22] Stimler, D.M., "Scheduling Turbofan Engine Control Set Points by Semi-infinite Optimization", Automatica, Vol. 25, No. 3, pp 413, 1989.

[23] Wittenmark Bjorn, "Integrators, Nonlinearities, and Anti- reset Windup for Different Control Structures", 1989 American Control Conference, Pittsburgh, PA, p1679.

[24] Astrom, K.J., Rundqwist L., "Integrator Windup and How to Avoid It", 1989 American Control Conference, Pittsburgh, PA, p1693.

[25] Kapasouris, P., Athans M., and Stein G., "Design of Feedback Control Systems for Stable Plants with Saturating Actuators", 27th IEEE Conf. on Dec. and Contr., Austin, Texas, 1988.

[26] Soeder J.F., "F100 Multivariable Control Synthesis Program", NASA TP 2231, Oct. 1983.

[27] Brown H. Elgen J.A., "Aircraft Engine Control Mode Analysis", ASME $84-\mathrm{GT}-262$.

[28] Emami-Naeini A., Khraishi N.M., Vincent J.H., Berg D.F., "Propulsion System Design Specifications Based on STOVL Flight Control Requirements", AIAA-90-3227, Aircraft Design, Systems and Operations Conference, Sept., 17, 1990. 


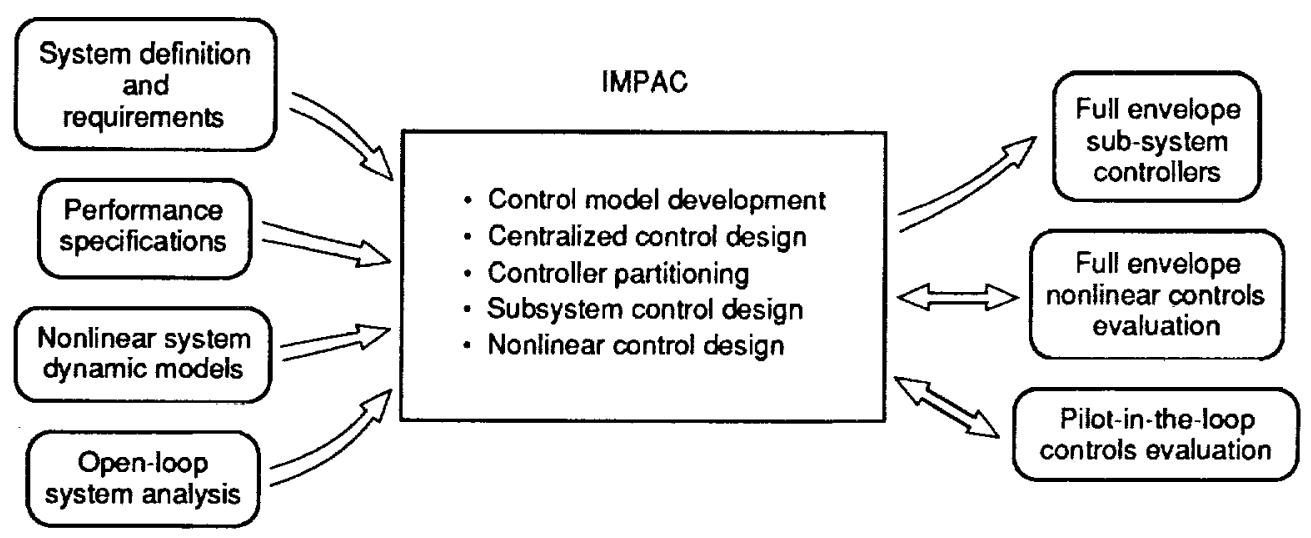

Figure 1. -IMPAC control design methodology.

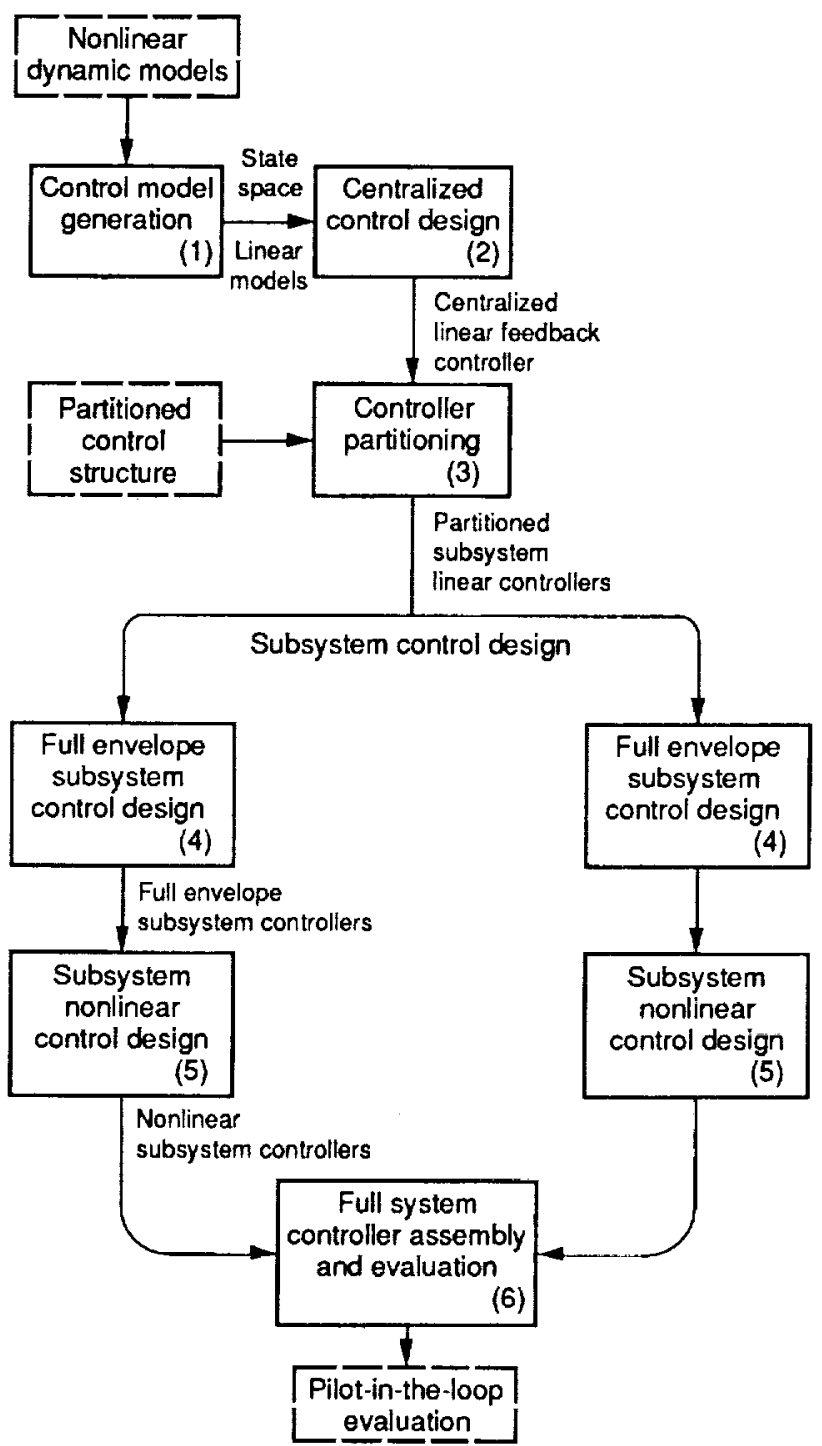

Figure 2.-IMPAC methodology flowchart. 


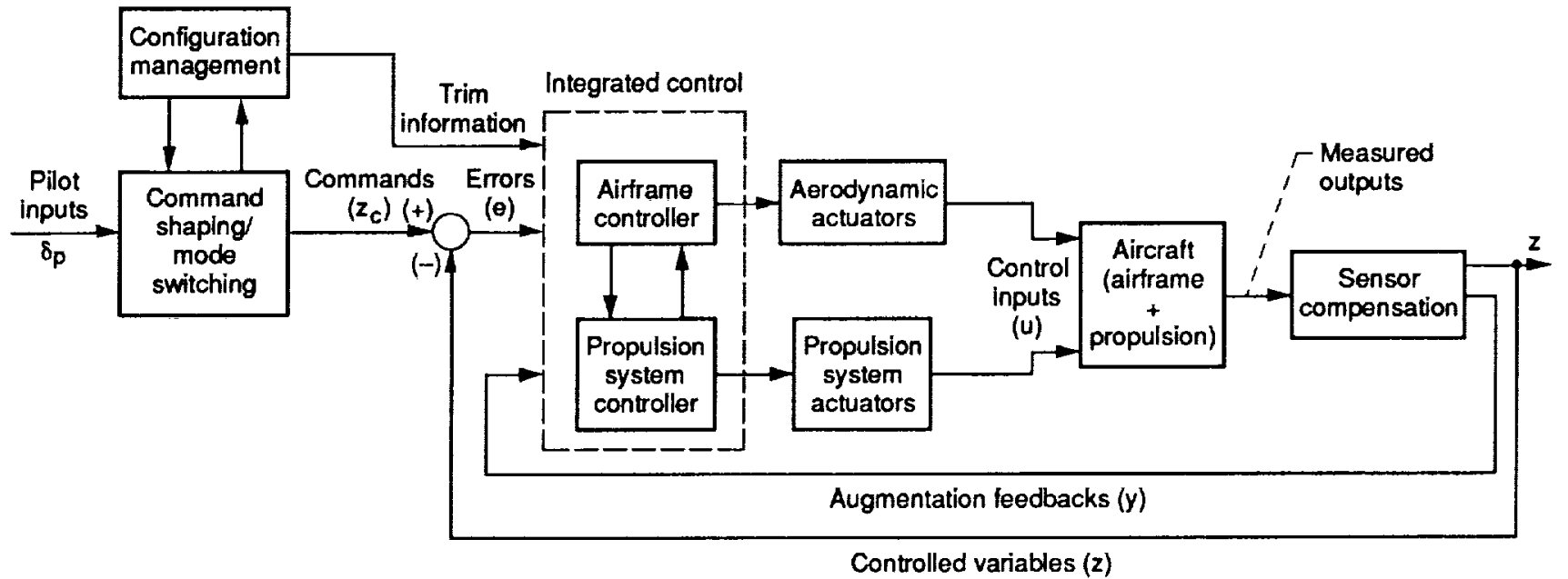

Figure 3.-Block diagram showing IFPC elements.

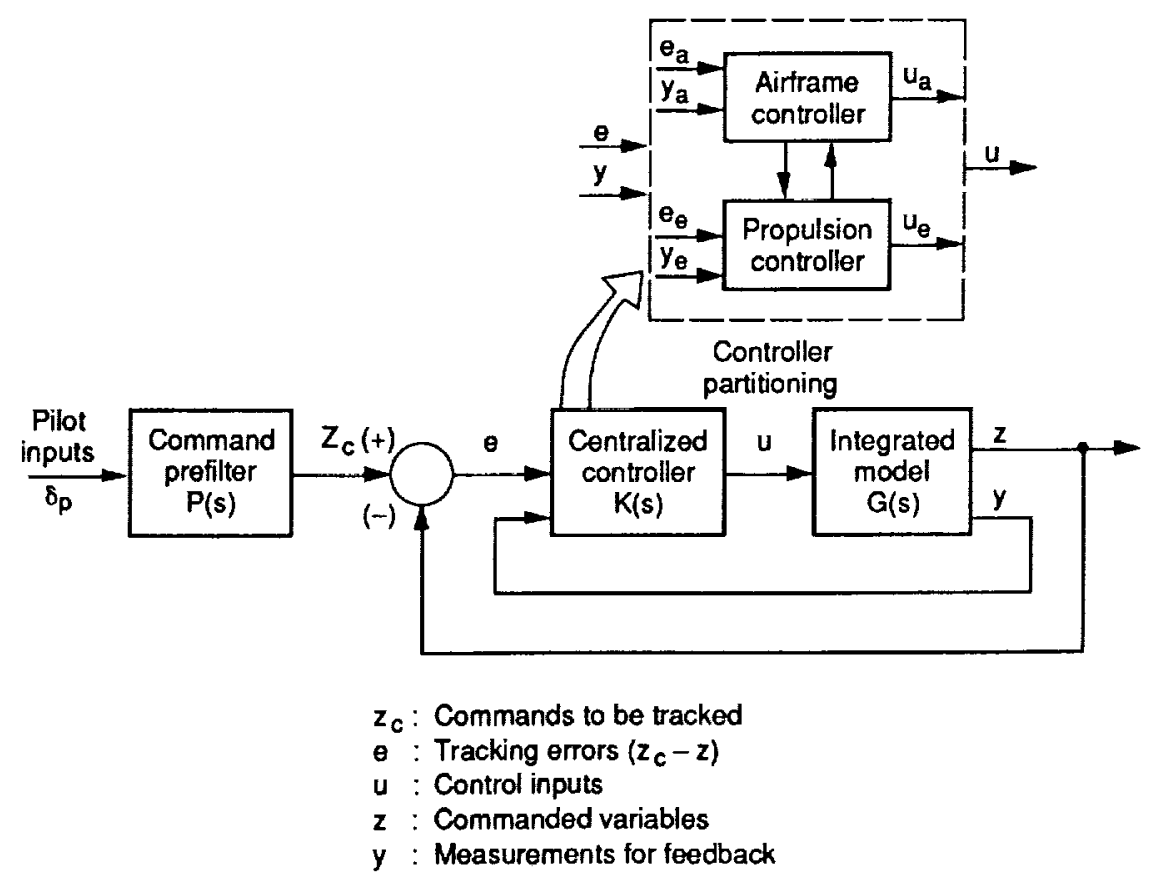

Figure 4.-Block diagram for linear control design. 


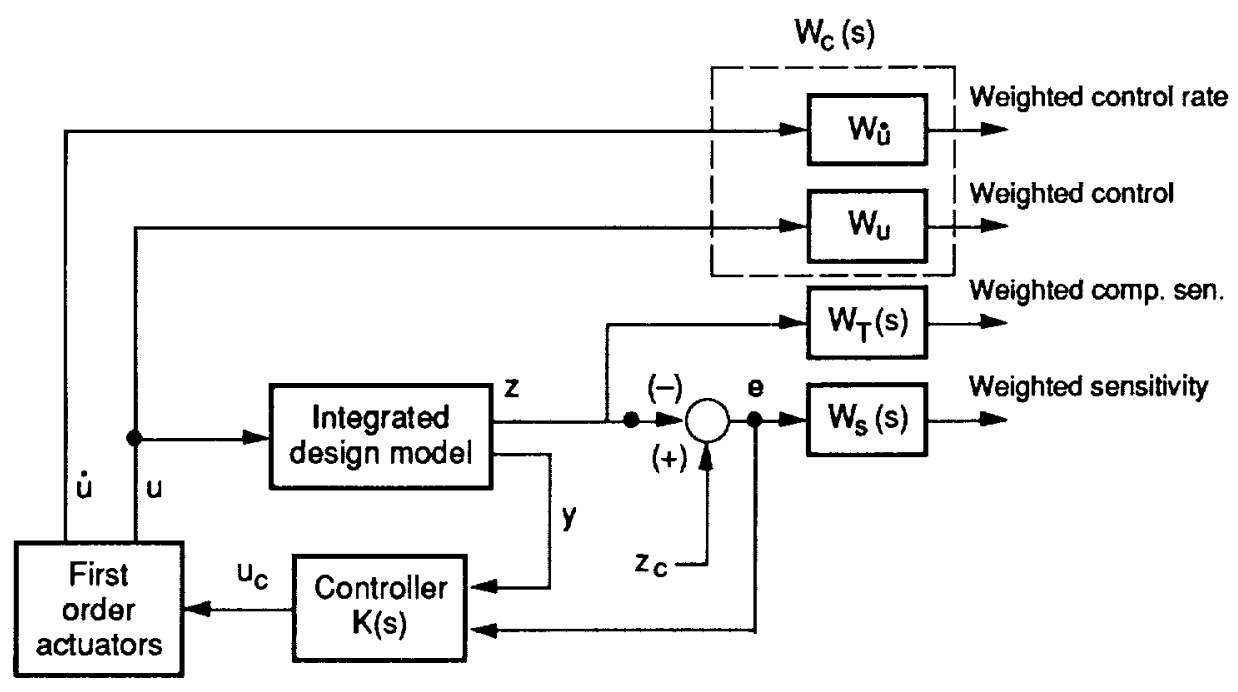

Figure 5.-Block diagram for $\mathrm{H}_{\infty}$ formulation of IFPC feedback design.

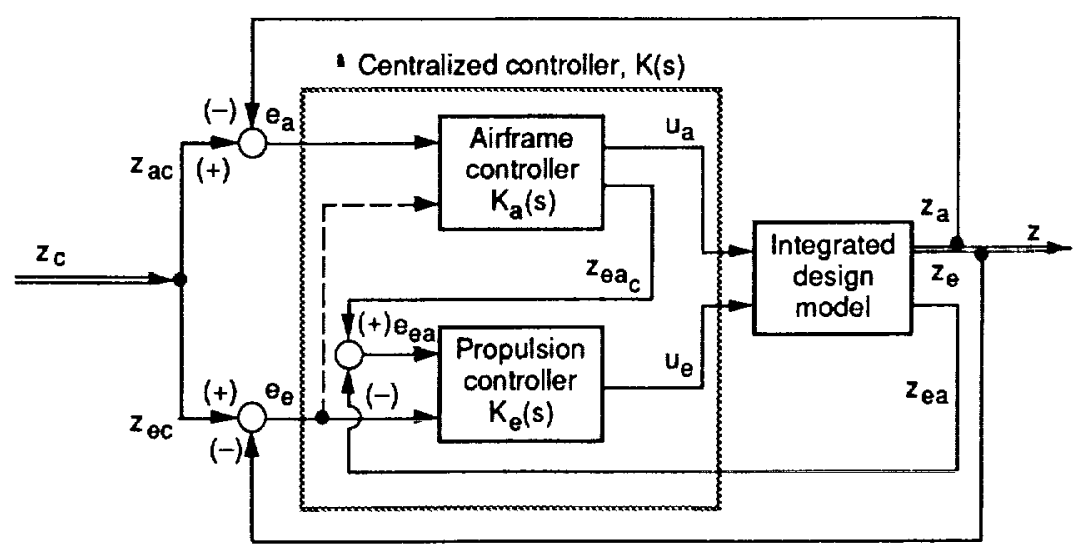

Figure 6. -Block diagram for decentralized, hierarchical controller partitioning. 


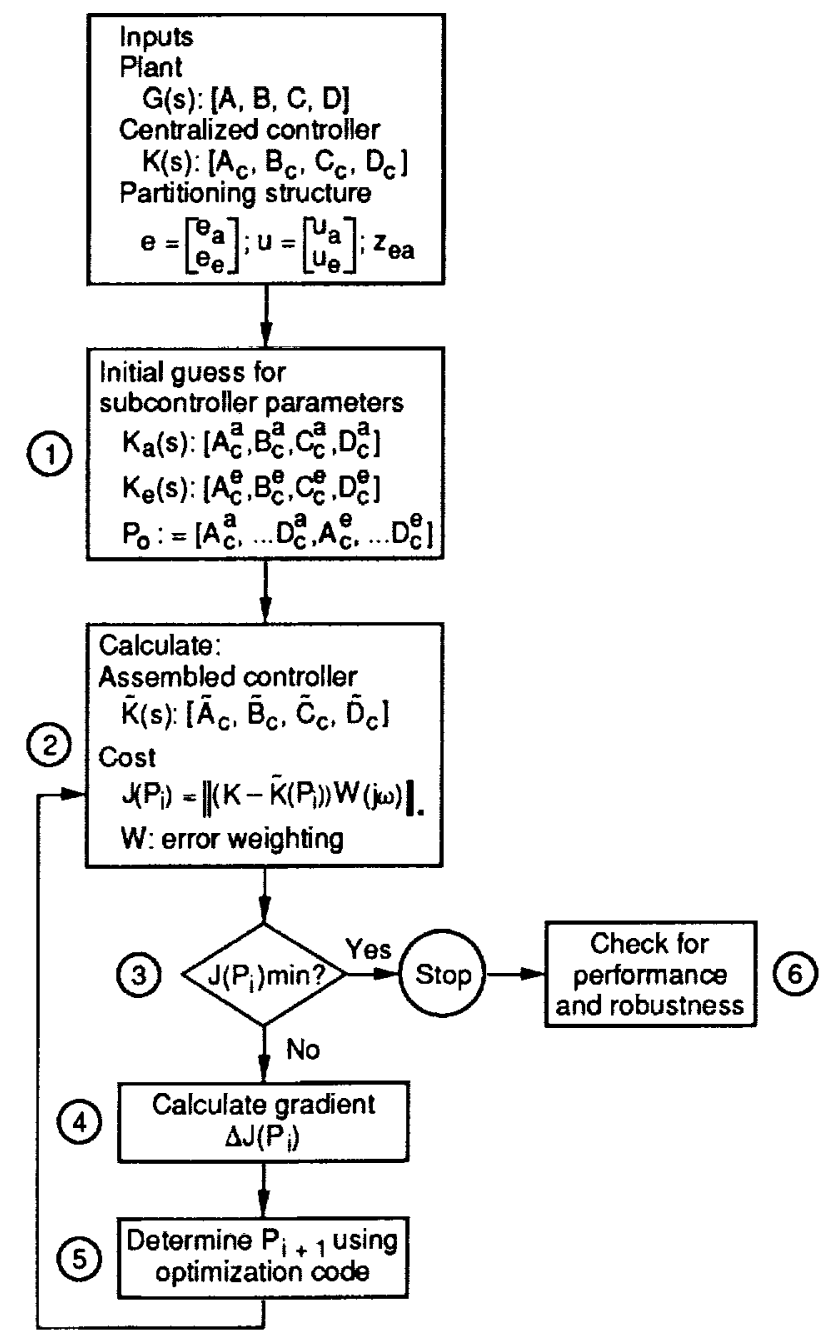

Figure 7.-Flow chart for controller positioning.

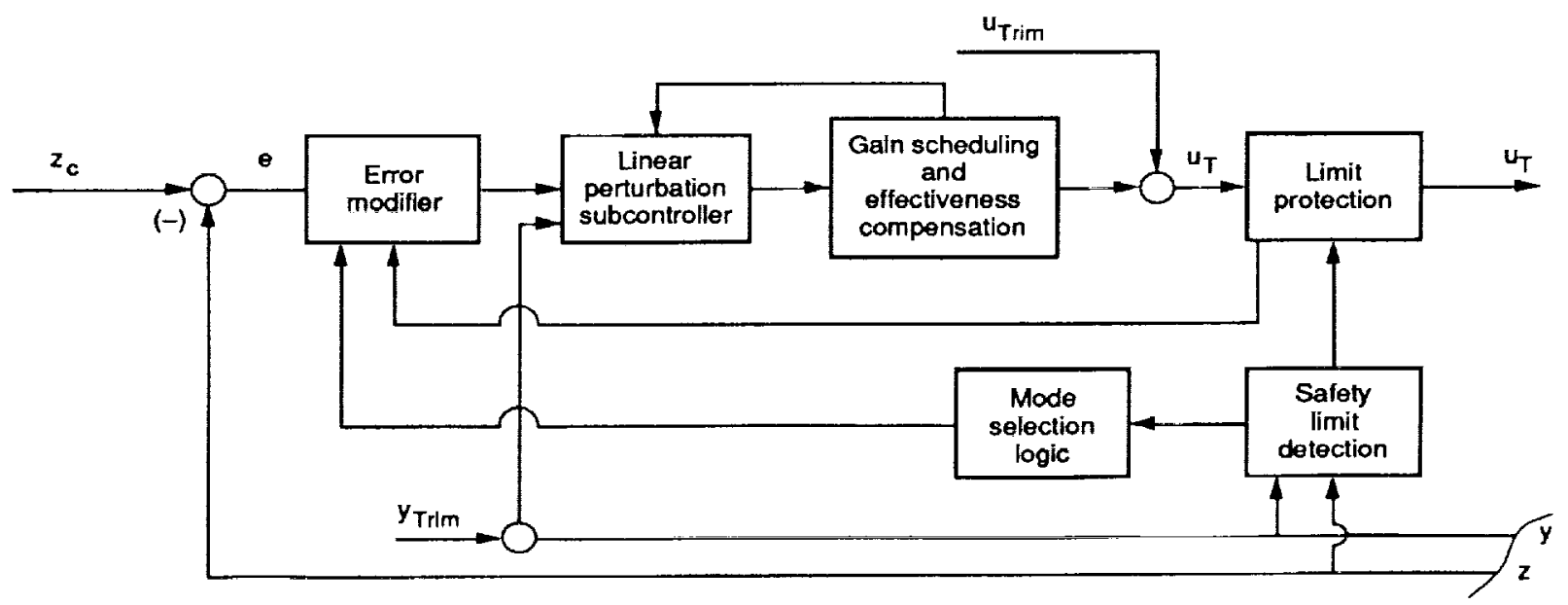

Figure 8.-A nonlinear subcontroller implementation. 


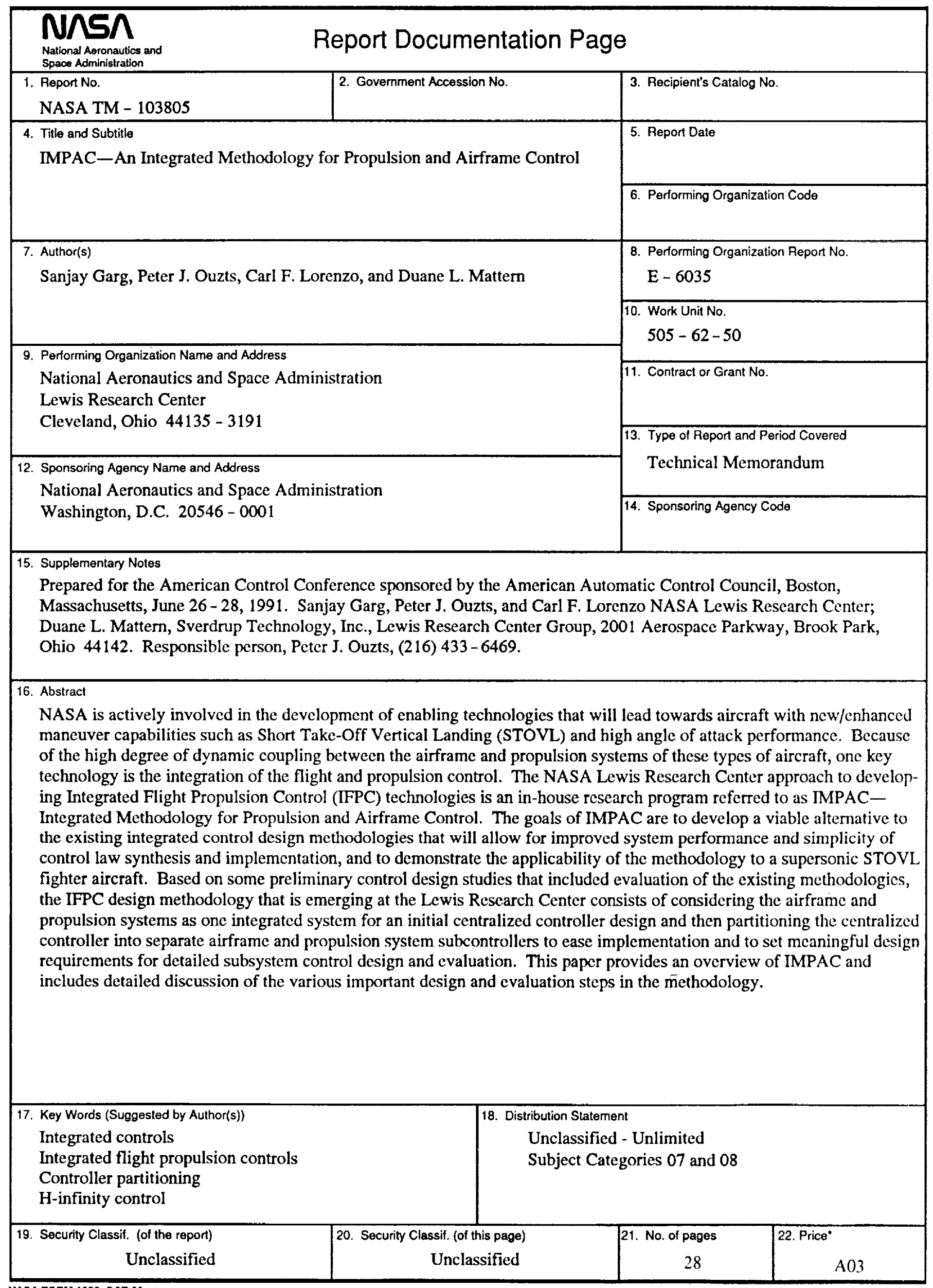


